\title{
Malnutrition Predisposes to Endotoxin-Induced Edema and Impaired Inflammatory Response in Parenterally Fed Piglets
}

Baek, Ole; Fabiansen, Christian; Friis, Henrik; Ritz, Christian; Koch, Jørgen; Willesen, Jakob L; Heegaard, Peter M. H.; Lykkesfeldt, Jens; Briend, André; Golden, Michael H

Total number of authors:

12

Published in:

Journal of Parenteral and Enteral Nutrition

Link to article, DOI:

10.1002/jpen.1705

Publication date:

2020

Document Version

Peer reviewed version

Link back to DTU Orbit

Citation (APA):

Baek, O., Fabiansen, C., Friis, H., Ritz, C., Koch, J., Willesen, J. L., Heegaard, P. M. H., Lykkesfeldt, J., Briend, A., Golden, M. H., Sangild, P. T., \& Thymann, T. (2020). Malnutrition Predisposes to Endotoxin-Induced Edema and Impaired Inflammatory Response in Parenterally Fed Piglets. Journal of Parenteral and Enteral Nutrition, 44(4), 668-676. https://doi.org/10.1002/jpen.1705

\section{General rights}

Copyright and moral rights for the publications made accessible in the public portal are retained by the authors and/or other copyright owners and it is a condition of accessing publications that users recognise and abide by the legal requirements associated with these rights.

- Users may download and print one copy of any publication from the public portal for the purpose of private study or research.

- You may not further distribute the material or use it for any profit-making activity or commercial gain

- You may freely distribute the URL identifying the publication in the public portal 
See discussions, stats, and author profiles for this publication at: https://www.researchgate.net/publication/335759113

\section{Malnutrition Predisposes to Endotoxin-Induced Edema and Impaired Inflammatory Response in Parenterally Fed Piglets}

Article in Journal of Parenteral and Enteral Nutrition · September 2019

DOI: 10.1002/jpen.1705

CITATIONS

3

12 authors, including:

Henrik Friis

University of Copenhagen

363 PUBLICATIONS 8,650 CITATIONS

SEE PROFILE

Jakob L Willesen

University of Copenhagen

62 PUBLICATIONS 1,134 CITATIONS

SEE PROFILE
READS

71

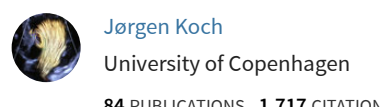

84 PUBLICATIONS 1,717 CITATIONS

SEE PROFILE

Jens Lykkesfeldt

University of Copenhagen

276 PUBLICATIONS 8,861 CITATIONS

SEE PROFILE

Some of the authors of this publication are also working on these related projects:

Project $\quad$ For those of you who have asked what my current project is: I am just helping others with their work. View project

Project NEOMUNE View project 


\section{Malnutrition Predisposes to Endotoxin-Induced Edema and Impaired Inflammatory Response in Parenterally Fed Piglets}

\author{
Ole Bæk, MD ${ }^{1, *} \mathbb{D}$; Christian Fabiansen, PhD $^{2, *}$; Henrik Friis, $\mathbf{P h D}^{2}$; \\ Christian Ritz, $\mathbf{P h D}^{2}$; Jørgen Koch, $\mathbf{P h D}^{3}$; Jakob L. Willesen, $\mathbf{P h D}^{3}$; \\ Peter M.H. Heegaard, $\mathrm{PhD}^{4, \dagger}$; Jens Lykkesfeldt, $\mathrm{DSc}^{1}$; André Briend, $\mathrm{PhD}^{2,5}$; \\ Michael H. Golden, $\mathrm{PhD}^{6}$; Per T. Sangild, $\mathrm{DSc}^{1}$; and Thomas Thymann, $\mathrm{PhD}^{1}$
}

WILEY

\begin{abstract}
Background: Poor nutrition status is common among hospitalized children and children in low-income countries and may be associated with increased susceptibility to edema and infections. We hypothesized that poor nutrition status, established with a suboptimal composition of parenteral nutrition (PN), predisposes to endotoxemia-induced edema, oxidative stress, and dysregulated immune responses. Methods: Using a $2 \times 2$ factorial design, 3-day-old piglets $(\mathrm{n}=40)$ were given either optimal or suboptimal composition of PN for 7 days and then infused with either saline or lipopolysaccharide (LPS) for 9 hours to induce an acute-phase reaction. Abdominal tissue edema and blood markers of immunity, inflammation, and oxidative stress were assessed. Results: Piglets receiving suboptimal nutrition showed signs of malnutrition with restricted growth, signs of inflammation (elevated C-reactive protein [CRP], interleukin-6, and serum amyloid A levels), oxidative stress (lower erythrocyte glutathione/hemoglobin and $\alpha$-tocopherol/cholesterol ratios), and liver dysfunction (increased liver weight and blood bilirubin levels). Perirenal edema was more excessive in malnourished LPS-infused animals, relative to healthy LPS-infused control animals $(P<.01)$. Malnutrition reduced the inflammatory response to LPS (lower CRP, tumor necrosis factor- $\alpha$, haptoglobin, and neutrophil to lymphocyte ratio) but did not influence LPS-induced oxidative stress markers. Conclusions: We conclude that endotoxemia and malnutrition in combination lead to acute-phase hyporesponsiveness and perirenal edema in piglets. This finding may have implications for pediatric patients that suffer from malnutrition, as their response to bacterial infections may differ substantially from patients of normal nutrition status. (JPEN J Parenter Enteral Nutr. 2019;0:1-9)
\end{abstract}

\title{
Keywords
}

edema; inflammation; kwashiorkor; lipopolysaccharide; malnutrition; oxidative stress; piglets

\footnotetext{
From the ${ }^{1}$ Department of Veterinary and Animal Science, University of Copenhagen, Copenhagen, Denmark; ${ }^{2}$ Department of Nutrition, Exercise and Sports, University of Copenhagen, Copenhagen, Denmark; ${ }^{3}$ Department of Veterinary Clinical Sciences, University of Copenhagen, Copenhagen, Denmark; ${ }^{4}$ Innate Immunology Group, National Veterinary Institute, Technical University of Denmark, Copenhagen, Denmark; ${ }^{5}$ University of Tampere School of Medicine and Tampere University Hospital, University of Tampere, Tampere, Finland; and the ${ }^{6}$ Department of Medicine and Therapeutics, University of Aberdeen, Aberdeen, UK.

*These authors should be considered co-first authors.

${ }^{\dagger}$ Present address: Department of Biotechnology and Biomedicine, Technical University of Denmark.
}

Financial disclosure: The research was supported by the University of Copenhagen, Denmark, and Nutriset, France. Fresenius Kabi, Sweden, donated ingredients for PN solutions. None of the contributors had any influence on the study design or execution, data interpretation, or preparation the manuscript.

Conflicts of interest: None declared.

Received for publication March 25, 2019; accepted for publication August 17, 2019.

This article originally appeared online on $\operatorname{xxxx} 0,2019$.

Corresponding Author:

Thomas Thymann, PhD, Department of Veterinary and Animal Science, University of Copenhagen, 68 Dyrlægevej, DK-1958 Frederiksberg C, Copenhagen, Denmark.

Email: thomas.thymann@sund.ku.dk 


\section{Clinical Relevancy Statement}

Critically or chronically ill children often have catabolism, leading to progressive malnutrition. The nutrition regimen, whether parenteral or enteral, is known to influence not only the degree of catabolism but also the susceptibility to infections. In turn, malnutrition increases the risk of serious infections and may alter the acute-phase response relative to patients of more optimal nutrition status. One such example is from low-income countries where malnutrition and infections often coincide and can associate with development of peripheral edema, as seen in kwashiorkor patients. This paper shows how endotoxemia-induced edema and acutephase responses depend on nutrition status in parenterally fed pigs.

\section{Introduction}

Edema is frequently observed during infections and malnutrition in pediatric patients. Generalized edema can be seen in children suffering from severe acute malnutrition (SAM) ${ }^{1}$ whereas this is rarely seen in other patient types - for example, short-bowel syndrome or other malabsorption disorders that can lead to malnutrition. ${ }^{2}$ In low-income countries, malnutrition is a known risk factor for development of infectious diseases, such as pneumonia, diarrhea, or sepsis. ${ }^{3-5}$ In some cases, children with SAM develop generalized edema - that is, kwashiorkor ${ }^{6}$ - which is associated with a much higher mortality and poorer response to nutrition treatment. ${ }^{7,8}$ The kwashiorkor syndrome also includes skin lesions, hypoalbuminemia, fatty liver, cholestasis, and electrolyte disturbances ${ }^{6,9}$ and may be at higher risk of endotoxemia because of small-bowel bacterial overgrowth and increased gut permeability. ${ }^{10,11}$ Although edema formation in kwashiorkor is often attributed entirely to hypoalbuminemia, there may an additive effect of noxious insults, such as infections, producing increased levels of free radicals that exceed the antioxidant capacity. ${ }^{12}$ Oxidative damage to membranes, glycocalyx, and extracellular matrix molecules associated with capillary function may lead to edema by decompartmentalization of water into the extracellular space. ${ }^{13}$

On this background, we hypothesized that a combination of induced malnutrition and gram-negative infection predisposes to edema and abdominal fluid accumulation as well as increased levels of oxidative stress. We specifically investigated the effects of systemic lipopolysaccharide (LPS) exposure on edema formation, inflammation, and oxidative stress in an animal model of induced malnutrition.

\section{Materials and Methods}

\section{Animals and Surgical Procedures}

The animal experiment was approved by the Danish Animal Experiments Inspectorate, Ministry of Food, Agriculture and Fisheries, Danish Veterinary and Food Administration (Permission: 2009/561-1731), which is in accordance with the guidelines from Directive 2010/63/EU of the European Parliament. Female piglets that were 43 days old (Landrace $\times$ Yorkshire $\times$ Duroc, Gadbjerg, Denmark) were separated from their mother and fasted for 3 hours before being anesthetized with an intramuscular injection $(0.1 \mathrm{~mL} / \mathrm{kg})$ of zolazepam/tiletamin (Zoletil $50 \mathrm{mg} / \mathrm{mL}$; Virbac, Kolding, Denmark), xylazine (Narcoxyl $20 \mathrm{mg} / \mathrm{mL}$, MSD Animal Health, Ballerup Denmark), ketamine (Ketaminol $100 \mathrm{mg} / \mathrm{mL}$, MSD Animal Health), and butorphanol (Torbugesic $10 \mathrm{mg} / \mathrm{mL}$, ScanVet, Fredensborg, Denmark). Antimicrobial prophylaxis was applied prior to surgery (Cephazolin, $20 \mathrm{mg} / \mathrm{kg}$, Fresenius Kabi, Germany). The ventral part of the neck was shaved, and local subcutaneous anesthesia was applied (Lidocain, 2\%). An incision was made and the internal jugular vein catheterized using silastic tubing (inner diameter $0.2 \mathrm{~mm}$ ). The catheters were tunneled subcutaneously with a trocar to the dorsal part of the neck, where they were exteriorized and connected to an infusion pump via a swivel system to allow free movement. The animals were housed individually in boxes with sawdust bedding and free access to drinking water. Boxes were placed on a heated tabletop, ensuring uniform heating for all pigs. After recovery from surgery, the animals were allocated to 2 groups (each $n=20$ ), ensuring equal average body weights between the groups. The experiment consisted of 2 interventions in a 2-phased design. In phase 1, from day 1 to 7 , the pigs were fed either nutritionally optimal (OPT) or suboptimal (MAL) parenteral nutrition (PN). In phase 2, on day 8, 2 groups were subdivided based on body weight to be co-infused with either LPS or saline (Figure 1). The 2 experimental diets were designed with substantial differences in macronutrient and micronutrient composition but with equal concentrations of sodium. The OPT group received PN nutritionally optimized for lean body mass growth in pigs, whereas the MAL group received an experimental PN containing less amino acid ( $5.5 \mathrm{vs} 47 \mathrm{~g} / \mathrm{L})$ and more fat (34.1 vs $9.7 \mathrm{~g} / \mathrm{L}$ ), with equal fractions of polyunsaturated fatty acids (PUFAs) and more carbohydrate (97.4 vs $84.5 \mathrm{~g} / \mathrm{L}$ ) and without addition of minerals and vitamins. The calculated energy levels were 736 vs $626 \mathrm{kcal} / \mathrm{L}$ in MAL vs OPT. The PN solutions were tailor-made using commercially available products (Kabiven, Vitalipid, Soluvit, Tracel, Vamin, all Fresenius Kabi, Bad Homburg, Germany), isotonic saline, and sterile water. The PN carbohydrate fraction was based on dextrose, and lipid fraction was Intralipid. For a full description of 2 PN solutions, see Supplementary Table S2. PN was initiated with an infusion rate of $6 \mathrm{~mL} / \mathrm{kg} / \mathrm{h}$, increasing to $8 \mathrm{~mL} / \mathrm{kg} / \mathrm{h}$ on day 2 . On day 4 , a concentrated iron solution ( $1 \mathrm{~mL}$ Hyofer $20 \%$, Virbac, Kolding, Denmark) was administered subcutaneously to all pigs to prevent anemia. In phase 2, the animals were coinfused for 9 hours with either LPS (lyophilized Escherichia 


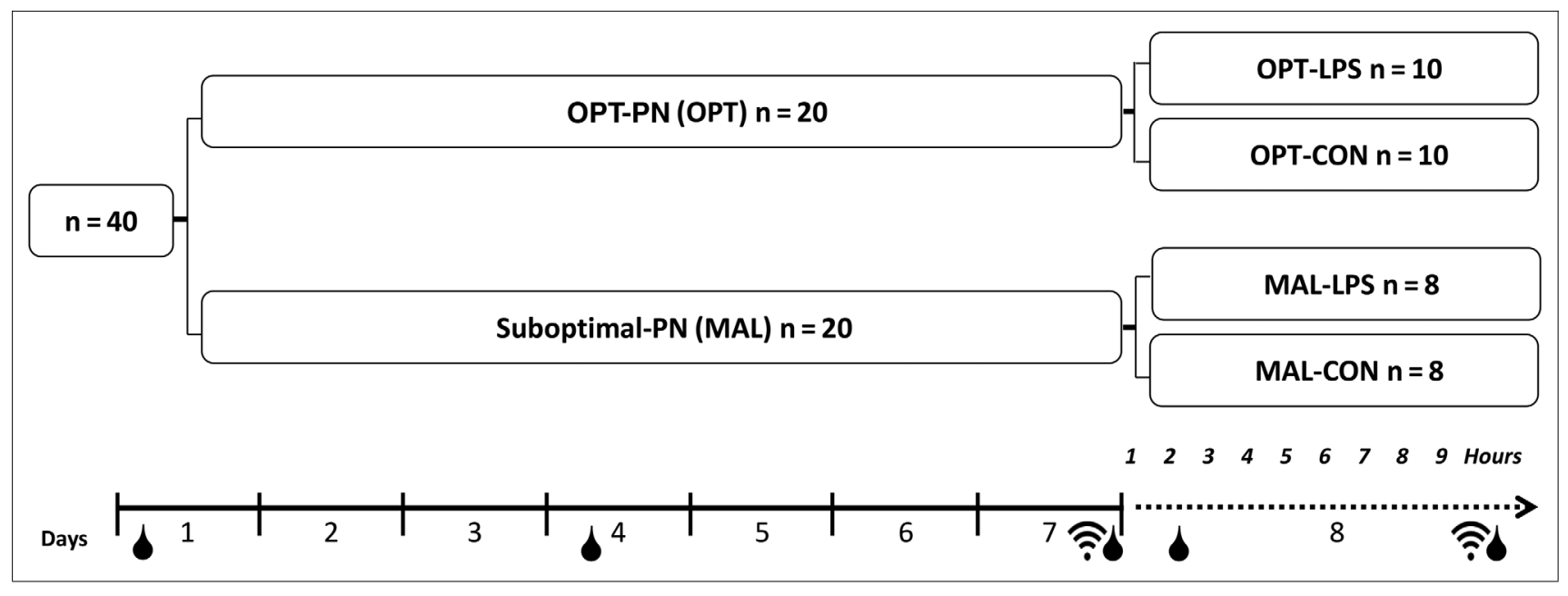

Figure 1. Experimental overview. Following baseline blood samples, animals were allocated to either OPT or MAL PN group for 7 days. Four animals died in the MAL group within the first 7 days. On day 8, half of the animals from each group were given LPS (OPT-LPS and MAL-LPS) for 9 hours, whereas the other half served as controls (OPT-CON and MAL-CON). A "droplet" indicates timing for drawing blood and a "wave" indicates timing of abdominal ultrasound. CON, control, LPS, lipopolysacharide, MAL, malnourished group, OPT, optimal group, PN, parenteral nutrition.

coli serotype 0111-B4, Sigma, \#L-2630; OPT-LPS $\mathrm{n}=$ 10 and MAL-LPS $\mathrm{n}=8$ ) or saline (OPT-CON, $\mathrm{n}=10$, and MAL-CON, $\mathrm{n}=8$ ). LPS was added directly to the PN solution, which continued to be infused at $8 \mathrm{~mL} / \mathrm{kg} / \mathrm{h}$, resulting in an LPS infusion rate of $10 \mu \mathrm{g} / \mathrm{kg} / \mathrm{h}$.

\section{Clinical Recordings, Blood Biochemistry, and Hematology}

Weight, temperature, and clinical status, based on a clinical score (Supplementary Table S1), were recorded daily and at 1 and 2 hours after initiation of LPS infusion. Blood samples were collected via the jugular catheter on day 1, 4, and 7 and subsequently 2 and 9 hours after start of LPS infusion (Figure 1). Blood collected at day 7 served as the end of phase 1 and baseline for phase 2. Blood for bloodgas analysis was collected in heparin-coated syringes and analyzed immediately on an automated blood-gas analyzer (GEM premier 3000, Lexington, KY, USA). Blood for hemogram and biochemical parameters were collected in heparin-coated vials. Biochemical analysis was performed on an Advia 1800 Chemistry System (Siemens Healthcare Diagnostics, Tarrytown, NY, USA). Hematological analysis was performed on an Advia 2120 hematology system (Siemens Healthcare Diagnostics).

Blood for measurement of immune and oxidative stress endpoints in plasma were collected in EDTA-containing tubes. Following separation, erythrocytes were isolated and sample aliquots were stored at $-80^{\circ} \mathrm{C}$ until further analysis.

\section{Edema Detection}

Abdominal ultrasonography using a Vivid I ultrasonographic system with a microconvex 8 C-RS (4.7-11 MHz) transducer (GE Healthcare, Brøndby, Denmark) was targeted to estimate fluid accumulation, performed just before the start of LPS infusion, and repeated just before euthanasia (Figure 1). All animals were examined in right lateral recumbency using gentle manual restrain without sedation. A general survey of the abdomen was performed with the following protocol: swipe-scanning with multiple planes of all quadrants assessing the urinary bladder, small intestine, right and left kidneys, spleen, and liver. Occurrence of free intra-abdominal fluid was recorded when found. With its well-defined anatomical borders, we used the width of the perirenal space as a quantifiable surrogate marker of edema. Perirenal fluid accumulation was measured as the maximum thickness of the perirenal anechoic rim in millimeters. Two calipers were placed between the outer layer of kidney and outer part of the anechoic zone. All images were stored digitally, and data analysis was performed using Echo PAC for PC, v7.0 (GE Healthcare, Brøndby, Denmark), in a blinded manner by a single individual. Prior to euthanasia, all animals were inspected visually for signs of peripheral edema, focusing on the extremities and abdomen and periorbitally. At the end of the 9-hour LPS infusion, the animals were anesthetized, a final blood sample was taken, and then animals were euthanized with an intracardiac injection of sodium pentobarbital $(60 \mathrm{mg} / \mathrm{kg})$.

\section{Oxidative Stress Markers}

Plasma vitamin E ( $\alpha$ - and $\gamma$-tocopherol) was analyzed by reversed-phase high-performance liquid chromatography as described by Sattler et al. ${ }^{14}$ Plasma malondialdehyde (MDA) was measured as described in detail elsewhere. ${ }^{15}$ Erythrocyte glutathione (GSH) was quantified using the 
method of Hissin and Hilf ${ }^{16}$ followed by correction for hemoglobin concentration as measured by a commercial kit according to the manufacturer's instructions (Cayman Chemical Company \#700540, Ann Arbor, MI, USA).

\section{Immune Markers}

Porcine interleukin-6 (IL-6) and tumor necrosis factor$\alpha(\mathrm{TNF}-\alpha)$ plasma concentrations were determined by enzyme-linked immunosorbent assay (ELISA; R\&D Systems: Duoset DY686 and Invitrogen: Swine TNF- $\alpha$ cytoset, CSC1753, respectively), following the manufacturers' instructions. Samples were run in duplicates in a dilution of $1: 2$ with a detection limit of $62.5 \mathrm{pg} / \mathrm{mL}$ for IL-6 and $31.3 \mathrm{pg} / \mathrm{mL}$ for TNF- $\alpha$. Plasma levels of haptoglobin were determined by ELISA using an in-house mouse antiporcine haptoglobin monoclonal antibody and a commercial rabbit anti-human haptoglobin detection antibody (DAKO A0030, DAKO, Glostrup, Denmark), as previously described. ${ }^{17}$ Detection limit was $13 \mu \mathrm{g} / \mathrm{mL}$. Plasma Creactive protein (CRP) was analyzed by ELISA, as described earlier, ${ }^{18}$ with a detection limit of $0.35 \mu \mathrm{g} / \mathrm{mL}$. Serum amyloid A (SAA) was determined using ELISA (Phase SAA assay, Tridelta Development, Kildare, Ireland) according to the manufacturer's instructions, with a detection limit of $31.3 \mu \mathrm{g} / \mathrm{mL}$.

\section{Statistical Analysis}

Analyses were carried out using the statistical software package Stata v12 (Stata/IC) (StataCorp, College station, TX, USA). Data from 2 phases of the study were analyzed separately. For phase 1, biochemical, oxidative stress, and immune markers were evaluated using linear mixed-effects models. These included a time-treatment interaction with the constraint of a single baseline level ${ }^{19}$ as fixed effect and pig-specific random effects to account for repeated measurements. Post hoc comparisons by means of $t$-tests were then made between the 2 treatment groups. For analysis of the same markers in phase 2, similar models with time-treatment interactions were used, except that groupdependent baseline levels were assumed to reflect the effects of the treatment in phase 1. Organ weights were analyzed by one-way analysis of variance (ANOVA). Post hoc comparisons by means of $t$-tests were used to assess the effects of LPS in the OPT and MAL groups. The combined effect of LPS and nutrition status on perirenal fluid accumulation was evaluated by one-way ANOVA. Immune parameters below the limit of detection were handled in the analysis by substitution the value with a value that was half the value for limit of detection. Model confirmation was based on residual and normal probability plots. Right-skewed outcomes were logarithm-transformed, and $P$-values $<.05$ were considered significant.
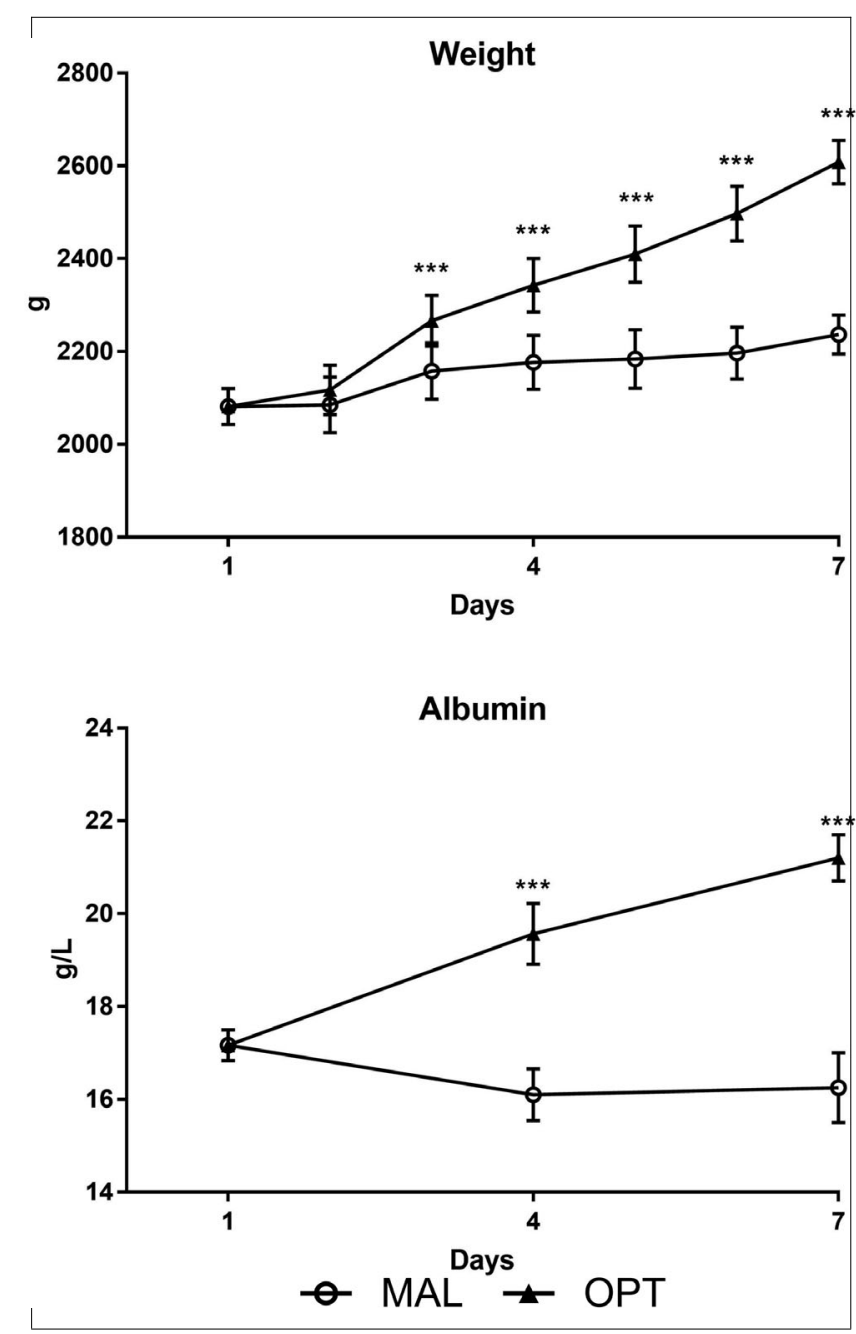

Figure 2. Development of body weight and plasma albumin level during phase 1 (day 1-7). $\mathbf{\Delta}$, OPT; ○, MAL. Data are shown as means with corresponding SEM. ${ }^{* * *} P<.001$. MAL, malnourished group; OPT, optimal group; SEM, standard error of the mean.

\section{Results}

\section{Phase 1}

Body weight and plasma albumin level were markedly reduced in MAL pigs (Figure 2), who also had an elevated clinical deterioration score ( 1.6 vs $1.1, P<.001)$ and higher rectal temperature $\left(39.6\right.$ vs $\left.38.4^{\circ} \mathrm{C}, P<.001\right)$ compared with OPT piglets. Four pigs in the MAL group died during phase $1 ; 1$ was euthanized because of catheter dislocation, another because of poor clinical condition, and 2 were found dead with necropsy indicating generalized pneumonia. The MAL pigs also had higher plasma CRP, IL-6, and SAA but lower haptoglobin and erythrocyte GSH/hemoglobin ratio (Figure 3). Surprisingly, however, in view of the higher PUFA intake, plasma MDA was also 


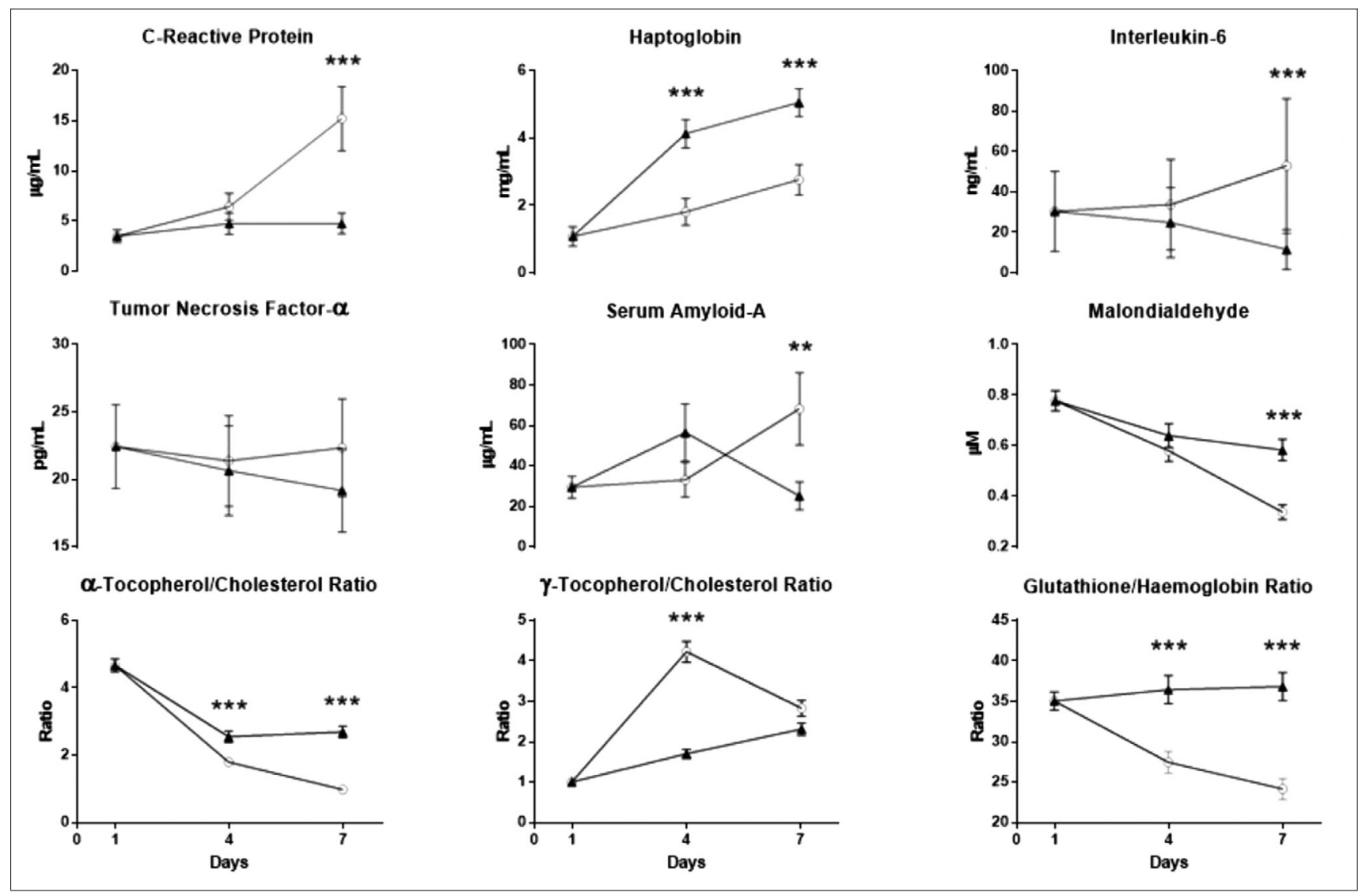

Figure 3. Development of immune and oxidative stress markers during phase 1 (day 1, 4, 7). $\mathbf{\Delta}$, OPT; $\circ$, MAL. Data are shown as means with corresponding SEM. ${ }^{* * *} P<.001$. MAL, malnourished group; OPT, optimal group; SEM, standard error of the mean.

lower in MAL pigs. The plasma $\alpha$-tocopherol/cholesterol ratio was reduced in the MAL pigs, whereas the serum $\gamma$-tocopherol/cholesterol ratio was increased (Figure 3). Although serum albumin levels were lower in MAL vs OPT at the end of the 7-day period (Figure 2, $P<.001$ ), this was opposite for globulin levels, which were higher in MAL (Supplementary Table S3, $P<.001$ ), resulting in similar total plasma protein in both groups (Supplementary Table S3, $P=.50$ ). Also, bilirubin levels were higher in MAL, whereas aspartate amino transferase was lower (both in Supplementary Table S3, both $P<.01$ ). Finally, abdominal ultrasound measurements at the end of phase 1 showed no fluid accumulation in any animal.

\section{Phase 2}

Both OPT-LPS and MAL-LPS piglets were clinically affected by LPS treatment, as indicated by an increased clinical deterioration score $(+0.5, P<.001)$ and increased rectal temperature $\left(+0.9^{\circ} \mathrm{C}, P<.001\right)$. Whereas generalized signs of edema were not observed in any piglets, the ultrasound measurements of the abdomen of OPT-LPS and MAL-LPS showed fluid accumulation in the perirenal space. However, MAL-LPS pigs showed markedly increased perirenal fluid accumulation relative to OPT-LPS pigs $(3.6$ vs $0.6 \mathrm{~mm}$, $P<.01$, Figure 4). Moreover, there were signs of abdominal free fluid in 2 of 8 piglets in the MAL-LPS groups and in no pigs from other groups. For organ weights, there was a main effect of LPS on relative kidney weight, as both LPS groups showed increased kidney weights relative to their control groups (Table $1, P<.05$ ). LPS did not affect the weight of other organs, except for liver and distal small intestine, in which LPS reduced the weights in MAL-LPS pigs and increased weights in OPT-LPS pigs (Table 1, both $P<.05$ ).

In contrast to organ weights, LPS infusion had multiple nutritionally dependent and independent effects on immune and oxidative stress markers. These are presented in Table 2 as mean values at the end of LPS infusion ( $t=9$ hours) with corresponding percent changes relative to baseline $(t$ $=0$ hours). After 9 hours, CRP levels were higher in the MAL-LPS group compared with OPT-LPS pigs (30.3 vs $13.5 \mathrm{mmol} / \mathrm{L}$ ). Because of higher baseline levels in MAL pigs, LPS led to less CRP increase in the MAL-LPS group relative to the OPT-LPS group $(57 \%$ vs $233 \%, P<.01)$. 


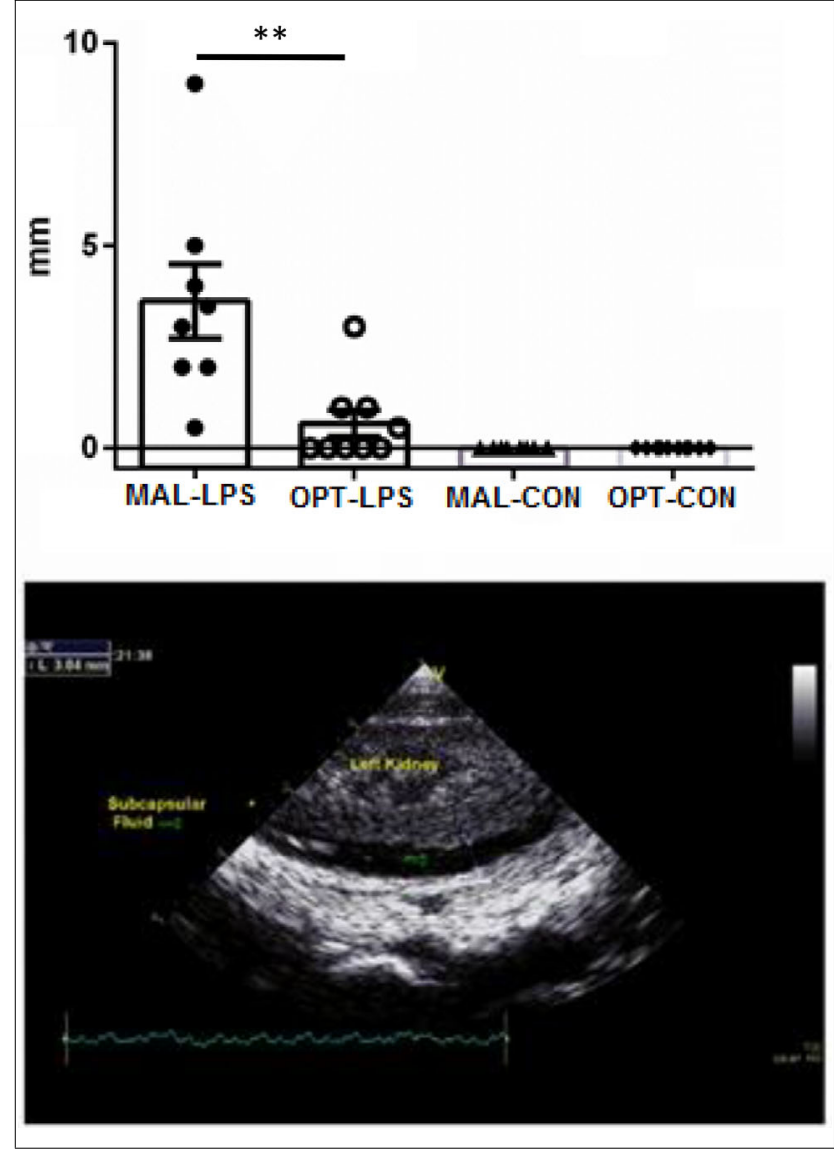

Figure 4. Perirenal fluid accumulation at the end of phase 2. Ultrasound image showing fluid in the perirenal space. Data are shown as mean width of the perirenal space with corresponding SEM. Included is an ultrasound image showing fluid in the perirenal space. ${ }^{* *} P<.01$. CON, control; LPS, lipopolysaccharide; MAL, malnourished group; OPT, optimal group; SEM, standard error of the mean; PN, parenteral nutrition.

Interestingly, there was a hyporesponsiveness in the MALLPS group with regard to TNF- $\alpha$, as the levels were much lower than those in OPT-LPS (70 vs $287 \mathrm{pg} / \mathrm{mL}, P<.05)$. The differences in TNF- $\alpha$ responses were most obvious after 2 hours, at which levels in MAL-LPS were much lower than those in OPT-LPS pigs (312 vs $7048 \mathrm{pg} / \mathrm{mL}$, $P<.001$, data not shown). Likewise, MAL-LPS pigs showed hyporesponsiveness with regard to haptoglobin $(2.4$ vs $5.6 \mathrm{mmol} / \mathrm{L}, P<.05)$ compared with OPT-LPS, whereas malnourishment did not affect the LPS SAA and IL-6 responses. The leucocyte subsets showed few effects of LPS. However, lymphocytes were unchanged in MAL-LPS but dropped in OPT-LPS (Supplementary Table S4, $P<.05$ ), resulting in an increased ratio of neutrophils to lymphocytes in OPT-LPS. Platelet counts, mean platelet volume and mean platelet component (MPC), at the end of LPS infusion were not presented as 16/38 samples coagulated during euthanasia and transport to laboratory, potentially skewing the results toward lower platelet counts.

The level of $\gamma$-tocopherol was higher in MAL-LPS vs OPT-LPS pigs (1.6 vs $0.4 \mu \mathrm{mol})$, with a significantly larger decrease in OPT-LPS $(-32 \%$ vs $-43 \%, P<.001)$. Independent of nutrition regimen, LPS lowered $\alpha$-tocopherol levels to $\approx 47 \%$ relative to baseline, with a significant main effect that was still present when correcting for cholesterol $(P<.001)$. Blood MDA levels dropped in both MAL-LPS and OPT-LPS pigs ( $P<.05$, no interaction).

A marked change was seen in bilirubin, with lower levels in MAL-LPS than in OPT-LPS but, relative to baseline, a significantly lower increase in MAL-LPS compared with that in OPT-LPS (Supplementary Table S4, $P<.01$ ). Lactate increased in all groups, including the control animals, during the LPS infusion, but less relative to baseline in MAL-LPS vs OPT-LPS (Supplementary Table S4, $P<.05)$. LPS led to several smaller but significant nutritiondependent changes in alkaline phosphatase, creatinine, urea, cholesterol, iron, MPC, and potassium (Supplementary Table S4, all $P<.05)$. Likewise, there were nutritionindependent effects on serum albumin level, potassium, creatinine kinase, $\gamma$-glutamyltransferase, globulins, magnesium, phosphate, calcium, erythrocytes, hemoglobin, basophiles, and hematocrit (Supplementary Table S4, all $P<.05)$.

\section{Discussion}

Suboptimal nutrition supply led to clear signs of malnutrition including slow growth, low plasma serum albumin level, and other biochemical indices indicating cholestasis, low muscle mass, and metabolic acidosis. As a key finding, the combined effect of LPS and malnutrition led to edema formation with fluid accumulation in the perirenal space. Although the edema was evident using ultrasound, we did not observe macroscopic signs of edema, possibly because of the short duration of the LPS infusion. In addition, LPS led to increased relative kidney weight irrespective of PN treatment, indicating parenchymal fluid accumulation. The experiment strongly suggests that malnourishment is a risk factor for the development of edema during an episode of endotoxemia.

Before introduction of LPS, MAL pigs progressively developed higher rectal temperature and elevated blood CRP, SAA, and IL-6 levels. In contrast, TNF- $\alpha$ levels showed no difference between the groups, which is consistent with findings in children with SAM. ${ }^{20}$ Surprisingly, circulating haptoglobin showed an inverse pattern with higher levels in piglets receiving optimal nutrition. It has been shown that children with protein malnutrition have increased levels of haptoglobin. ${ }^{20-22}$ For oxidative stress parameters, we found lower levels of GSH and GSH/hemoglobin ratio in the malnourished animals, indicative of a higher level of 
Table 1. Organ Weights Relative to Body Weight.

\begin{tabular}{|c|c|c|c|c|c|c|}
\hline Organ weights & MAL-CON & MAL-LPS & OPT-CON & OPT-LPS & $\begin{array}{c}P \\
\text { Interaction }^{\mathrm{a}}\end{array}$ & $\begin{array}{c}P \\
\text { Main } \\
\text { Effect }^{b}\end{array}$ \\
\hline Brain, $\mathrm{g} / \mathrm{kg}$ & $17.1 \pm 0.8$ & $16.7 \pm 0.7$ & $14.9 \pm 0.6$ & $14.8 \pm 0.6$ & NS & NS \\
\hline Heart, g/kg & $8.4 \pm 1.1$ & $7.6 \pm 0.2$ & $7.9 \pm 0.4$ & $6.7 \pm 0.2$ & NS & NS \\
\hline Lungs, $\mathrm{g} / \mathrm{kg}$ & $19.5 \pm 1$ & $17.6 \pm 0.7$ & $17.9 \pm 0.8$ & $18.9 \pm 1.0$ & NS & NS \\
\hline Liver, $\mathrm{g} / \mathrm{kg}$ & $35.1 \pm 1.7$ & $30.4 \pm 1.4$ & $25.6 \pm 1.2$ & $27.6 \pm 1.2$ & $<.05$ & - \\
\hline Kidneys, g/kg & $8.6 \pm 0.6$ & $10.5 \pm 0.5$ & $8.6 \pm 0.7$ & $10.6 \pm 0.4$ & NS & $<.05$ \\
\hline Spleen, $\mathrm{g} / \mathrm{kg}$ & $4.7 \pm 1.3$ & $4.9 \pm 1.2$ & $3.3 \pm 1.1$ & $3.0 \pm 1.0$ & NS & NS \\
\hline Stomach, $\mathrm{g} / \mathrm{kg}$ & $6.2 \pm 0.3$ & $6.6 \pm 0.3$ & $6.7 \pm 0.1$ & $7.2 \pm 0.2$ & NS & NS \\
\hline Proximal Intestine, $\mathrm{g} / \mathrm{kg}$ & $5.7 \pm 0.2$ & $6.0 \pm 0.3$ & $6.7 \pm 0.4$ & $6.4 \pm 0.2$ & NS & NS \\
\hline Middle Intestine, $\mathrm{g} / \mathrm{kg}$ & $6.9 \pm 0.2$ & $6.8 \pm 0.3$ & $7.2 \pm 0.3$ & $7.4 \pm 0.3$ & NS & NS \\
\hline Distal Intestine, $\mathrm{g} / \mathrm{kg}$ & $8.0 \pm 0.2$ & $7.3 \pm 0.4$ & $8.6 \pm 0.4$ & $9.6 \pm 0.4$ & $<.05$ & - \\
\hline Colon, $\mathrm{g} / \mathrm{kg}$ & $7.9 \pm 0.4$ & $7.9 \pm 0.3$ & $8.4 \pm 0.6$ & $10.7 \pm 2.3$ & NS & NS \\
\hline
\end{tabular}

Data are presented as means $( \pm$ SEM). Significant effects $(P \leq 0.05)$ are shown. Where an interaction between LPS and PN group is present, no main effect of LPS is presented.

CON, control; Dist., distal; LPS, lipopolysaccharide; MAL, malnourished group; Mid., middle; NS, not significant; OPT, optimal group; PN, parenteral nutrition; Prox., proximal; SEM, standard error of the mean.

${ }^{a}$ LPS effect dependent on nutrition regimen: (OPT-LPS - OPT-CON) - (MAL-LPS - MAL-CON).

${ }^{b}$ LPS effect independent of nutrition regimen: (OPT-LPS + MAL-LPS) - (OPT-CON + MAL-CON).

oxidative stress. Surprisingly though, we also found lower levels of MDA, yet the absolute levels of MDA in both groups were within the normal range and thus too low to indicate any substantial oxidative stress. Furthermore, given the higher intake of PUFAs in the MAL group, the lower levels of MDA are unexpected and indicate that it may be a poor marker of oxidative stress under these conditions. The serum $\alpha$-tocopherol/cholesterol ratio was lower in the MAL group, which could indicate an increased rate of antioxidant turnover. However, the level of serum $\gamma$-tocopherol relative to cholesterol was higher in MAL vs OPT, which presumably reflects the $\gamma$-tocopherol present in the lipid fraction of $\mathrm{PN}$, which was provided in higher amounts for the MAL group.

After the LPS challenge, we found nutrition-dependent differences in the TNF- $\alpha$ response, which was much weaker in malnourished pigs, with corresponding weaker responses for CRP and haptoglobin. However, it should be taken into account that CRP levels were already elevated in the MAL group, before LPS infusion. Lower TNF- $\alpha$ responses in malnourished mice have been reported, ${ }^{23}$ and release of other cytokines, most notably IL-1, is also reduced in children with kwashiorkor. ${ }^{20,24}$ Surprisingly, there were no significant nutrition-independent effects of LPS on IL-6 and SAA. The cellular inflammatory response showed few nutritiondependent effects of LPS. However, the lack of an increase in the neutrophil to lymphocyte ratio in MAL-LPS indicates a lack of immune activation. After initiation of LPS infusion, the only marker for oxidative stress that showed significant nutrition-dependent effects was $\gamma$-tocopherol, with a larger decrease in OPT pigs, which most likely is an indicator of increased antioxidant consumption. However, because of the higher intake of $\gamma$-tocopherol precursors in the suboptimal PN, these results should be interpreted with caution. Surprisingly, MDA levels significantly decreased during LPS infusion, which, together with the relatively low levels found during phase 1, could further indicate that MDA may not be a sufficiently sensitive marker for our setting. Thus, we found no convincing evidence of increased oxidative stress as an effect of LPS in the malnourished piglets and cannot correlate this to the observed edema. It is possible that the short duration of the malnutrition period was inadequate to deplete the antioxidant capacity of the pigs. Also, the higher level of $\gamma$-tocopherol supplementation in MAL may have offered some protection against oxidative stress.

There were several nutrition-dependent effects of LPS on the biochemical, hematological, and venous blood-gas markers. However, most of these were small and probably not biologically relevant. The most marked nutritiondependent effects of LPS were on bilirubin, creatinine, and lactate. Lactate increased in all groups, but with a particularly high increase in OPT-LPS pigs. Before the final blood sampling, all pigs were subjected to stressful handling, especially during abdominal ultrasound, which required manual restraint. This could also explain why we saw clear increases in creatinine kinase in 3 of 4 groups, corresponding to increased muscle contractions. The lower muscle mass in the MAL pigs may explain the differences between groups. The bilirubin increased in both LPS groups but more dramatically in OPT-LPS, which is unexpected because bilirubin is not an acute-phase reactant. However, LPS is known to induce cholestasis, ${ }^{25,26}$ which could lead to hepatic dysfunction. Our findings of dilated bile ducts in LPS animals support this. Independent of nutrition 
Table 2. Effects of LPS on Immune and Oxidative Stress Markers.

\begin{tabular}{|c|c|c|c|c|c|c|c|c|c|c|}
\hline & MAL-CON & $\Delta(\%)$ & MAL-LPS & $\Delta(\%)$ & OPT-CON & $\Delta(\%)$ & OPT-LPS & $\Delta(\%)$ & $\begin{array}{c}P \\
\text { Interaction }^{\mathrm{a}}\end{array}$ & $\begin{array}{c}P \\
\text { Main } \\
\text { Effect }^{\mathrm{b}}\end{array}$ \\
\hline \multicolumn{11}{|l|}{ Immune markers } \\
\hline $\mathrm{CRP}, \mu \mathrm{g} / \mathrm{mL}^{\mathrm{c}}$ & $32.4 \pm 8.1$ & +13 & $30.4 \pm 7.1$ & +57 & $8.3 \pm 3.1$ & +26 & $13.5 \pm 1.8$ & +233 & $<.01$ & - \\
\hline $\begin{array}{l}\text { Haptoglobin, } \\
\mathrm{mg} / \mathrm{mL}^{\mathrm{c}}\end{array}$ & $3.4 \pm 0.9$ & +11 & $2.4 \pm 0.8$ & +6 & $6.0 \pm 1.5$ & +32 & $5.6 \pm 0.5$ & +22 & $<.05$ & - \\
\hline IL-6, ng/mL ${ }^{\mathrm{c}}$ & $52.7 \pm 26.1$ & +81 & $112 \pm 54$ & -28 & $17.2 \pm 7.0$ & -53 & $45.1 \pm 29.4$ & -11 & NS & NS \\
\hline $\mathrm{SAA}, \mu \mathrm{g} / \mathrm{mL}^{\mathrm{c}}$ & $115 \pm 26$ & -13 & $180 \pm 60$ & +114 & $61.6 \pm 36.3$ & +8 & $72.4 \pm 23.4$ & +85 & NS & NS \\
\hline $\begin{array}{l}\mathrm{TNF}-\alpha \\
\mathrm{pg} / \mathrm{mL}^{\mathrm{c}}\end{array}$ & $22.5 \pm 6.8$ & -46 & $75.5 \pm 41.4$ & +45 & $21.9 \pm 6.3$ & -63 & $287 \pm 89$ & +454 & $<.05$ & - \\
\hline \multicolumn{11}{|c|}{ Oxidative stress markers } \\
\hline $\begin{array}{l}\alpha \text {-Tocopherol, } \\
\mu \mathrm{M}\end{array}$ & $3.2 \pm 0.7$ & -4 & $1.8 \pm 0.3$ & -47 & $3.6 \pm 0.4$ & +19 & $1.6 \pm 0.1$ & -47 & NS & $<.001$ \\
\hline $\begin{array}{c}\alpha \text {-Tocopherol: } \\
\text { cholesterol }\end{array}$ & $1.3 \pm 0.1$ & -4 & $1.1 \pm 0.1$ & -18 & $2.9 \pm 0.1$ & -4 & $2.7 \pm 0.1$ & -10 & NS & $<.001$ \\
\hline $\begin{array}{c}\gamma \text {-Tocopherol, } \\
\mu \mathrm{M}^{\mathrm{c}}\end{array}$ & $2.2 \pm 0.4$ & -1 & $1.6 \pm 0.2$ & -32 & $1.0 \pm 0.1$ & +18 & $0.4 \pm 0$ & -43 & $<.001$ & - \\
\hline $\begin{array}{c}\gamma \text {-Tocopherol: } \\
\text { cholesterol }\end{array}$ & $1.0 \pm 0.1$ & +4 & $1.1 \pm 0.1$ & -15 & $0.8 \pm 0.1$ & -3 & $0.7 \pm 0.1$ & -4 & NS & NS \\
\hline $\mathrm{GSH}, \mu \mathrm{M}^{\mathrm{c}}$ & $90 \pm 5$ & +13 & $76 \pm 1$ & +31 & $130 \pm 7$ & +20 & $133 \pm 11$ & +69 & NS & NS \\
\hline $\mathrm{GSH} / \mathrm{HbG}$ & $24.5 \pm 2.4$ & -9 & $23.7 \pm 2.7$ & -1 & $41.6 \pm 2.9$ & +10 & $40 \pm 2.8$ & +8 & NS & NS \\
\hline $\mathrm{MDA}, \mu \mathrm{M}$ & $0.42 \pm 0.06$ & +6 & $0.39 \pm 0.05$ & -17 & $0.48 \pm 0.05$ & -22 & $0.38 \pm 0.03$ & -44 & NS & $<.05$ \\
\hline
\end{tabular}

Data are presented as means ( \pm SEM), with percentile changes $(\Delta)$ from baseline to end of LPS infusion. Significant effects $(P \leq .05)$ are shown. Where an interaction between LPS and PN group is present, no main effect of LPS is presented.

CON, control; CRP, C-reactive protein; GSH, glutathione; HbG, hemoglobin; IL-6, interleukine-6; LPS, lipopolysaccharide; MAL, malnourished group; MDA, malondialdehyde; NS, not significant; OPT, optimal group; PN, parenteral nutrition; SAA, serum amyloid A; SEM, standard error of the mean; TNF- $\alpha$, tumor necrosis factor- $\alpha$.

${ }^{a}$ LPS effect dependent on nutrition regimen: (OPT-LPS - OPT-CON) - (MAL-LPS - MAL-CON).

${ }^{b}$ LPS effect independent of nutrition regimen: (OPT-LPS + MAL-LPS) - (OPT-CON + MAL-CON).

${ }^{\mathrm{c} C}$ Calculations on a log-transformed scale, means transformed back, SEM presented are based on non-log-transformed data.

regimen, LPS infusion led to a decrease in erythrocytes and hematocrit, indicating a plasma volume expansion. It is possible that plasma volume expansion may be concealing part of the expected oxidative stress response.

The PN solutions used were purely experimental and did not reflect a clinical setting. However, we were able to induce a phenotype mimicking malnourished children within 7 days, and the lack of enteral stimulation has some similarities to children receiving PN. The edema formation in the malnourished group may share some pathological traits with children with kwashiorkor and septic patients with some degree of malnutrition. Biochemically, the similarities with kwashiorkor were less obvious; we found signs of cholestasis and an increase in plasma iron in the MAL group, but potassium and sodium were unaffected. In future experiments, it would be relevant to apply a more protracted exposure to LPS to see if more of the phenotypical traits of kwashiorkor develop over time.

\section{Conclusion}

This study found that the combined effect of poor nutrition status and endotoxemia led to a clear accumulation of fluid in the perirenal space. This was further associated with a weaker inflammatory response including a reduced CRP, haptoglobin, and TNF- $\alpha$ response. This could explain the increased mortality and morbidity from infections seen in malnourished children. We could not, however, show a clear correlation between nutrition status, edema formation, and markers of oxidative stress or antioxidant capacity. We used a very standardized experimental approach to identify the interactive effects of nutrition status and LPS-induced inflammation, and although the pathological response does not entirely mimic the edema development seen in patients suffering from kwashiorkor, the data do suggest that nutrition status and endotoxemia in combination may be important to edema development. More protracted studies in malnourished piglets exposed to low-dose LPS should be conducted to further test this hypothesis.

\section{Statement of Authorship}

T. Thymann, P. T. Sangild, and C. Fabiansen equally contributed to the conception and design of the research; $\mathrm{M}$. M. Golden and A. Birend contributed to the design of the research; C. Fabiansen, H. Friis, J. Koch, J. L. Willesen, 
P. M. H. Heegaard, and J. Lykkesfeldt contributed to the acquisition and analysis of the data; O. Bæk and C. Ritz contributed to the interpretation of the data; and O. Bæk and C. Fabiansen drafted the manuscript. All authors critically revised the manuscript, agree to be fully accountable for ensuring the integrity and accuracy of the work, and read and approved the final manuscript.

\section{Acknowledgments}

We thank the staff at the Section of Comparative Pediatric and Nutrition, University of Copenhagen, for valuable technical assistance.

\section{Supplementary Information}

Additional supporting information may be found online in the Supporting Information section at the end of the article.

\section{References}

1. WHO. UNICEF-WHO-The World Bank: Joint child malnutrition estimates-levels and trends. World Health Organization; 2016.

2. Cole CR, Frem JC, Schmotzer B, et al. The rate of bloodstream infection is high in infants with short bowel syndrome: relationship with small bowel bacterial overgrowth, enteral feeding, and inflammatory and immune responses. J Pediatr. 2010;156(6):941-947.e1. https://doi.org/10.1016/j.jpeds.2009.12.008

3. Olofin I, McDonald CM, Ezzati M, et al. Associations of suboptimal growth with all-cause and cause-specific mortality in children under five years: a pooled analysis of ten prospective studies. PLoS One. 2013;8(5):e64636. https://doi.org/10.1371/journal.pone.0064636

4. Caulfield LE, de Onis M, Blössner M, Black RE. Undernutrition as an underlying cause of child deaths associated with diarrhea, pneumonia, malaria, and measles. Am J Clin Nutr. 2004;80(1):193-198. http://www.ncbi.nlm.nih.gov/pubmed/15213048. Accessed January 25, 2017.

5. Maitland K, Berkley JA, Shebbe M, Peshu N, English M, Newton CRJC. Children with severe malnutrition: can those at highest risk of death be identified with the who protocol? PLoS Med. 2006;3(12):e500. https://doi.org/10.1371/journal.pmed.0030500

6. Williams C. Kwashiorkor: a nutritional disease of children associated with a maize diet. Lancet. 1935;226(5855):1151-1152. https://doi.org/ 10.1016/S0140-6736(00)94666-X

7. Prudhon C, Golden MH, Briend A, Mary JY. A model to standardise mortality of severely malnourished children using nutritional status on admission to therapeutic feeding centres. Eur J Clin Nutr. 1997;51(11):771-777.

8. Schofield C, Ashworth A. Why have mortality rates for severe malnutrition remained so high? Bull World Health Organ. 1996;74(2):223-229.

9. Wellcome Trust Working Party. Classification of infantile malnutrition. Lancet. 1970;2(7667):302-303.

10. Crane RJ, Jones KDJ, Berkley JA. Environmental enteric dysfunction: an overview. Food Nutr Bull. 2015;36(1_suppl1):S76-S87. https://doi.org/10.1177/15648265150361S113

11. Klein K, Fuchs GJ, Kulapongs P, Mertz G, Suskind RM, Olson RE. Endotoxemia in protein-energy malnutrition. $J$ Pediatr
Gastroenterol Nutr. 1988; 7(2):225-228. http://www.ncbi.nlm.nih.gov/ pubmed/3127570. Accessed September 29, 2017.

12. Golden MHN, Ramdath D. Free radicals in the pathogenesis of kwashiorkor. Proc Nutr Soc. 1987;46(1):53-68. https://doi.org/ 10.1079/PNS19870008

13. Henry Golden M. Nutritional and other types of oedema, albumin, complex carbohydrates and the interstitium - a response to Malcolm Coulthard's hypothesis: Oedema in kwashiorkor is caused by hypo-albuminaemia. Paediatr Int Child Health. 2015;35(2):90-109. https://doi.org/10.1179/2046905515Y.0000000010

14. Sattler W, Mohr D, Stocker R. Rapid isolation of lipoproteins and assessment of their peroxidation by high-performance liquid chromatography postcolumn chemiluminescence. Methods Enzymol. 1994;233:469-489.

15. Lykkesfeldt J. Determination of malondialdehyde as dithiobarbituric acid adduct in biological samples by HPLC with fluorescence detection: comparison with ultraviolet-visible spectrophotometry. Clin Chem. 2001;47(9):1725-1727.

16. Hissin PJ, Hilf R. A fluorometric method for determination of oxidized and reduced glutathione in tissues. Anal Biochem. 1976;74(1):214-226.

17. Sorensen NS, Tegtmeier C, Andresen LO, et al. The porcine acute phase protein response to acute clinical and subclinical experimental infection with Streptococcus suis. Vet Immunol Immunopathol. 2006;113(12):157-168. https://doi.org/10.1016/j.vetimm.2006.04.008

18. Heegaard PMH, Pedersen HG, Jensen AL, Boas U. A robust quantitative solid phase immunoassay for the acute phase protein $\mathrm{C}$ reactive protein (CRP) based on cytidine 5'-diphosphocholine coupled dendrimers. J Immunol Methods. 2009;343(2):112-118. https://doi.org/ 10.1016/j.jim.2009.02.002.

19. Fitzmaurice GM, Laird NM, Ware JH. Applied Longitudinal Analysis. Hoboken, NJ: John Wiley \& Sons; 2012: 134.

20. Rytter MJH, Kolte L, Briend A, Friis H, Christensen VB. The immune system in children with malnutrition - a systematic review. PLoS One. 2014;9(8):e105017. https://doi.org/10.1371/journal.pone.0105017.

21. Razban SZ, Olusi SO, Ade-Serrano MA, Osunkoya BO, Adeshina $\mathrm{HA}$, McFarlane H. Acute phase proteins in children with proteincalorie malnutrition. J Trop Med Hyg. 1975;78(12):264-266. http://www.ncbi.nlm.nih.gov/pubmed/57243. Accessed January 4, 2017.

22. Suliman OSM, Salih MAM, Karrar ZA, Mohammed AO, Helsing C. Acute phase reactants in Sudanese children with severe protein-energy malnutrition. Sudan J Paediatr. 2011;11(1):48-59. http://www.ncbi. nlm.nih.gov/pubmed/27493306. Accessed January 4, 2017.

23. Fock RA, Vinolo MAR, de Moura Sá Rocha V, de Sá Rocha LC, Borelli P. Protein-energy malnutrition decreases the expression of TLR-4/MD-2 and CD14 receptors in peritoneal macrophages and reduces the synthesis of TNF- $\alpha$ in response to lipopolysaccharide (LPS) in mice. Cytokine. 2007;40(2):105-114. https://doi.org/10.1016/ j.cyto.2007.08.007

24. Bhaskaram P, Sivakumar B. Interleukin-1 in malnutrition. Arch Dis Child. 1986;61(2):182-185. http://www.ncbi.nlm.nih.gov/pubmed/ 3082298. Accessed January 16, 2017.

25. Geier A, Fickert P, Trauner M. Mechanisms of disease: mechanisms and clinical implications of cholestasis in sepsis. Nat Clin Pract Gastroenterol Hepatol. 2006;3(10):574-585. https://doi.org/10.1038/ ncpgasthep 0602

26. Trauner M, Fickert $\mathrm{P}$, Stauber RE. Inflammation-induced cholestasis. J Gastroenterol Hepatol. 1999;14(10):946-959. https://doi.org/ 10.1046/j.1440-1746.1999.01982.x 